\title{
A randomised clinical trial of the efficacy of drop squats or leg extension/leg curl exercises to treat clinically diagnosed jumper's knee in athletes: pilot study
}

\author{
L J Cannell, J E Taunton, D B Clement, C Smith, K M Khan
}

\begin{abstract}
Objectives-To compare the therapeutic effect of two different exercise protocols in athletes with jumper's knee.

Methods-Randomised clinical trial comparing a 12 week programme of either drop squat exercises or leg extension/leg curl exercises. Measurement was performed at baseline and after six and 12 weeks. Primary outcome measures were pain (visual analogue scale 1-10) and return to sport. Secondary outcome measures included quadriceps and hamstring moment of force using a Cybex II isokinetic dynamometer at $30 \%$ second. Differences in pain response between the drop squat and leg extension/curl treatment groups were assessed by 2 (group) $\times$ 3 (time) analysis of variance. Two by two contingency tables were used to test differences in rates of return to sport. Analysis of variance (2 (injured versus non-injured leg) $\times 2$ (group) $\times 3$ (time)) was also used to determine differences for secondary outcome measures.
\end{abstract}

Results-Over the 12 week intervention, pain diminished by 2.3 points $(36 \%)$ in the leg extension/curl group and 3.2 points $(57 \%)$ in the squat group. There was a significant main effect of both exercise protocols on pain $(p<0.01)$ with no interaction effect. Nine of 10 subjects in the drop squat group returned to sporting activity by 12 weeks, but five of those subjects still had low level pain. Six of nine of the leg extension/curl group returned to sporting activity by 12 weeks and four patients had low level pain. There was no significant difference between groups in numbers returning to sporting activity. There were no differences in the change in quadriceps or hamstring muscle moment of force between groups.

Conclusions-Progressive drop squats and leg extension/curl exercises can reduce the pain of jumper's knee in a 12 week period and permit a high proportion of patients to return to sport. Not all patients, however, return to sport by that time.

(Br $\mathcal{F}$ Sports Med 2001;35:60-64)

Keywords: knee; patellar tendon; tendinopathy; tendinosis; eccentric strengthening; strength training
The term jumper's knee ${ }^{1}$ refers to the clinically diagnosed condition of activity related anterior knee pain associated with tenderness at the inferior pole of the patella where the patellar tendon attaches. This condition has many other names such as patellar tendinitis and patellar tendinopathy. ${ }^{2}$ In this paper we use the term jumper's knee to refer to the clinical condition and patellar tendinopathy to refer to the histopathology underlying the condition. We avoid the term tendinitis when discussing overuse tendon conditions. ${ }^{3}$

Although numerous conservative ${ }^{4}$ and surgi$\mathrm{cal}^{5}$ treatments have been recommended for jumper's knee, none has provided a highly effective confident cure for this condition. ${ }^{6}$ Strengthening exercises have been recommended as a treatment for jumper's knee ${ }^{4-9}$ and other tendinopathies. ${ }^{71011}$ To our knowledge, there has been no prospective, controlled study evaluating the clinical outcome of strength exercises for jumper's knee. ${ }^{6}$ Drop squats have been recommended as a treatment for jumper's $\mathrm{knee}^{7}$ as have standard strength exercises-the leg extension and the leg curl. ${ }^{8}$

Therefore to determine the effectiveness of either drop squat exercises or a combined leg extension/leg curl programme in patients with jumper's knee, we undertook a randomised clinical trial comparing the effect of these exercises on pain, return to sporting activity, and muscle strength.

\section{Materials and methods}

STUDY DESIGN

The study design was a randomised controlled trial with a 12 week intervention of either drop squats or leg curl/extension exercises. Clinical evaluation and testing was performed at baseline and six and 12 weeks. Subjects were not asked to refrain from sporting activities once their initial symptoms were relieved. Research assistants blinded to subjects' treatment group collected data. The University of British Columbia human research ethics committee approved the study. All subjects provided written informed consent.

\section{SUBJECTS}

There were 19 subjects (13 men, six women) with clinically diagnosed jumper's knee. Their ages ranged from 15 to 50 years. The sports the subjects played were basketball (six), soccer (three), running (three), volleyball (two), tennis (one), squash (one), rowing (one), American football (one), and gymnastics 
Table 1 Protocol used by subjects in the drop squat group

\begin{tabular}{lllll}
\hline & \multicolumn{2}{l}{ Hand weights $(\mathrm{kg})$} & \\
\cline { 2 - 4 } Progression & $\begin{array}{l}\text { Body weight } \\
45-60 \mathrm{~kg}\end{array}$ & $\begin{array}{l}\text { Body weight } \\
61-75 \mathrm{~kg}\end{array}$ & $\begin{array}{l}\text { Body weight } \\
76-90 \mathrm{~kg}\end{array}$ & \multirow{2}{*}{ Activity level } \\
\hline Start & 0 & 0 & 0 & Modified rest \\
Level 1 & 2.3 & 2.3 & 4.5 & Jogging - alternate days \\
Level 2 & 4.5 & 4.5 & 4.5 & Half speed \\
Level 3 & 6.8 & 9.1 & 14 & Three quarter speed \\
Level 4 & 9.1 & 14 & 18 & Full speed daily running \\
\hline
\end{tabular}

Table 2 Protocol used by subjects for leg extension. Leg curl was performed with half this weight

\begin{tabular}{lllll}
\hline & \multicolumn{2}{l}{ Weights $(\mathrm{kg})$} & & \\
\cline { 2 - 4 } Progression & $\begin{array}{l}\text { Body weight } \\
45-60 \mathrm{~kg}\end{array}$ & $\begin{array}{l}\text { Body weight } \\
61-75 \mathrm{~kg}\end{array}$ & $\begin{array}{l}\text { Body weight } \\
76-90 \mathrm{~kg}\end{array}$ & \multirow{2}{*}{ Activity level } \\
\hline Start & 4.5 & 4.5 & 4.5 & Modified rest \\
Level 1 & 4.5 & 11 & 11 & Jogging - alternate days \\
Level 2 & 9.1 & 14 & 18 & Half speed \\
Level 3 & 11 & 18 & 25 & Three quarter speed \\
Level 4 & 14 & 23 & 32 & Full speed daily running \\
\hline
\end{tabular}

(one). None used orthotics, as this was an exclusion criterion. Fifteen subjects had unilateral knee pain (nine left, six right), and three had bilateral symptoms but left sided symptoms were significantly greater than right. Subjects were diagnosed by one of three experienced sports medicine doctors at our referral based university sports medicine centre. Diagnostic criteria were (a) sports related anterior knee pain well localised to the inferior pole of the patella and present for more than four weeks, $(b)$ no other knee symptoms currently or previous to this episode of jumper's knee, (c) moderate or severe tenderness to palpation at the patellar tendon insertion into the patella, and $(d)$ otherwise normal knee examination.

\section{EXERCISE PROTOCOLS}

Subjects were randomised by sealed envelope draw to progressive exercise training in one of two groups: a group that performed drop squat exercises (squat group, $\mathrm{n}=10$ ) or progressive training using leg extension/leg curl exercise (extension/curl group; $n=9$ ). Subjects in both groups were treated with ice, antiinflammatory medications, and relative rest in the first two weeks of the study. They were all trained in the correct technique by a single investigator (LJC).

The squat group performed three sets of 20 drops once a day five days a week. The drop squats were performed by subjects starting from a standing position and unlocking their knees rapidly and dropping until both thighs were just short of parallel to the ground. Subjects were advised to use the quadriceps muscles of both legs to stop their fall just short of the thighs being parallel to the ground. The aim of the drop squats was to obtain high eccentric loading. When the subject was able to do three sets of 20 drops easily, he/she progressed to the next level of work as shown in table 1 . Subjects were encouraged to ice their patellar tendons after performing the drop squat programme and to expect some pain.

Once the subject's knee pain was completely absent, he/she began an alternate day running programme beginning with $1 \mathrm{~km}$ in running athletes and increasing by $1 \mathrm{~km}$ every third run, with intensity increasing as shown in table 1 .

The leg extension/curl group performed three sets of 10 lifts for each of the leg extension and leg curl exercises, once a day five days a week. For leg extension, subjects were advised to slowly lift the weight with the injured leg to full extension and to hold the weight in that position for two seconds. Each lift from start to finish took at least five seconds. This lift was repeated up to 10 times (one set) before the subject rested. Subjects began with a $5 \mathrm{~kg}$ weight and gradually increased their repetitions until they could do three sets of 10 with that weight. Once this was achieved, subjects progressed according to the protocol in table 2 . The aim of the leg extension exercise, and also the leg curl described below, was to provide high concentric loading.

Subjects performed the isotonic hamstring curl with half of the weight used for leg extension (table 2). The subject lay prone on the table and slowly flexed the knee to $90^{\circ}$, held the knee flexed in this position for two seconds, and then slowly returned the weight to the starting position. Each lift from start to finish took at least five seconds. Subjects completed three sets of 10 repetitions with the same weight on each leg, once a day five days a week.

Once the subject's knee pain was completely absent, he/she began an alternate day running programme beginning with $1 \mathrm{~km}$ in running athletes and increasing by $1 \mathrm{~km}$ every third run, with intensity increasing as shown in table 2 .

PAIN AND RETURN TO SPORTING ACTIVITY

Pain in the injured leg was measured on a visual analogue scale from 1 to 10 : 1 denoted pain free and 10 denoted severe pain and disability. Return to sporting activity was recorded at 12 week measurement.

\section{MUSCLE STRENGTH}

Quadriceps and hamstring moment of force were evaluated in both legs using a Cybex II isokinetic dynamometer. Lever length and torque were recorded at $30 \%$ second. The instrument was calibrated before each session for each subject.

\section{STATISTICAL ANALYSIS}

Differences in pain response between the drop squat and leg extension/curl treatment groups were assessed by 2 (group) $\times 3$ (time) analysis of variance. Two by two contingency tables were used to test differences in rates of return to sport. Analysis of variance (2 (injured versus non-injured leg) $\times 2$ (group) $\times 3$ (time)) was also used to determine differences for each of the following: quadriceps moment of force, hamstring moment of force, and thigh girth. Results were considered significant at $\mathrm{p}<0.05$.

\section{Results}

Table 3 gives the baseline characteristics of the subjects in the two groups. All subjects had been treated for jumper's knee before entering the study but not in the preceding three months. There were no drop outs and all subjects completed at least 55 of the maximum 60 
Table 3 Descriptive variables at baseline

\begin{tabular}{lll}
\hline Variable at baseline & Drop squat group $(n=10)$ & $\begin{array}{l}\text { Leg extension/curl group } \\
(n=9)\end{array}$ \\
\hline Males: females & $7: 3$ & $6: 3$ \\
Age (years) & $26(23$ to 29$)$ & $26(19$ to 33$)$ \\
Weight $(\mathrm{kg})$ & $76(69$ to 83$)$ & $74(66$ to 82$)$ \\
Height $(\mathrm{cm})$ & $172(165$ to 179$)$ & $174(167$ to 181$)$ \\
Duration of symptoms (months) & $3.1(1.6$ to 4.6$)$ & $4.2(2.3$ to 6.1$)$ \\
\hline
\end{tabular}

Values are number or mean (95\% confidence interval).

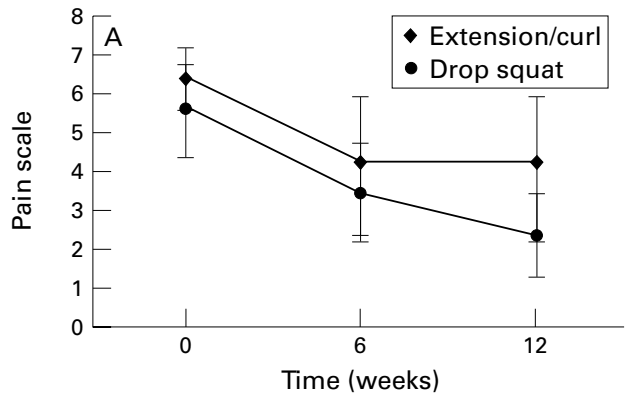

Figure 1 Knee pain scores. Mean scores with $95 \%$ confidence intervals are plotted at 0, 6, and 12 weeks for the drop squat group and the leg extension/curl group. There was a significant main effect for pain over time $(p<0.01)$ in both groups, but no interaction effect.

(five sessions a week) training sessions. Where possible, the chief investigator (LJC) observed the training session being performed by subjects in both groups.

\section{PAIN AND RETURN TO SPORT}

There was no difference in pain between the two treatment groups at baseline, but over the 12 week intervention there was a significant main effect for pain reduction in both groups $(p<0.01)$. Figure 1 shows plots of the individual scores. There was no significant interaction effect over the three time intervals, suggesting that trend to decreased pain was similar in the two treatment groups.

In the drop squat group, four subjects became completely pain free (two after six weeks, two after 12 weeks), five improved their pain scores (mean of 2.8 scale points), and one had an increase in pain score (3 scale points). In the leg extension/curl group, two subjects became pain free (one after six weeks, one after 12 weeks), four improved their pain scores (mean of 2.2 scale points), one remained

Table 4 Mean quadriceps moment of force

\begin{tabular}{llll}
\hline & 0 & 6 weeks & 12 weeks \\
\hline Injured leg: drop squats & $520(157)$ & $570(162)$ & $597(161)$ \\
Non-injured leg: drop squats & $620(171)$ & $678(218)$ & $654(192)$ \\
Injured leg: Ext/Curls & $620(114)$ & $591(121)$ & $591(131)$ \\
Non-injured leg: Ext/Curls & $684(180)$ & $694(113)$ & $656(126)$ \\
\hline
\end{tabular}

Values are expressed in newtons and are means (SD).

Ext, Extension.

Table 5 Mean hamstrings moment of force

\begin{tabular}{llll}
\hline & 0 & 6 weeks & 12 weeks \\
\hline Injured leg: drop squats & $271(123)$ & $286(114)$ & $309(122)^{\star}$ \\
Non-injured leg: drop squats & $282(111)$ & $293(107)$ & $312(108)^{\star}$ \\
Injured leg: Ext/Curls & $287(98)$ & $320(93)$ & $338(91)^{\star}$ \\
Non-injured leg: Ext/Curls & $300(88)$ & $328(90)$ & $332(85)^{\star}$ \\
\hline
\end{tabular}

Values are expressed in newtons and are means (SD).

$\star$ Significantly different from baseline, $\mathrm{p}<0.001$.

Ext, Extension. unchanged, and two had an increase in pain score (mean of 1.5 scale points). Nine of 10 subjects who performed the drop squat programme returned to sporting activity by 12 weeks, as did six of nine subjects who performed the leg extension/curl programme (no significant difference).

\section{MUSCLE STRENGTH}

Quadriceps moment of force was significantly $(\mathrm{p}<0.001)$ lower in the injured than the uninjured leg at baseline in both groups (table 4). There was no significant gain in quadriceps strength over the 12 week intervention in either group (table 4). Hamstring moment of force did not differ between injured and uninjured legs at baseline, and increased significantly $(\mathrm{p}<0.001)$ in both groups over the training period (table 5).

\section{Discussion}

To our knowledge, this is the first report of a randomised clinical trial of exercise treatment for jumper's knee-the condition of activity related anterior knee pain associated with tenderness at the junction of the patellar tendon with the inferior pole of the patella. In this discussion we focus on four clinically relevant issues: (a) the clinical diagnosis of this condition; (b) the effect of exercise treatment on pain; (c) factors associated with pain reduction; (d) study limitations and strengths.

\section{IS CLINICAL DIAGNOSIS OF JUMPER'S KNEE} JUSTIFIED?

In the late 1980s and early 1990s, ultrasound (US) was shown to accurately image tendon morphology, so it was hoped that this would correlate with symptoms and thus aid medical management of patients with tendon conditions. Unfortunately, despite the early optimism, ${ }^{12}{ }^{13}$ neither US ${ }^{14}$ nor magnetic resonance imaging $(\mathrm{MRI})^{15}$ has proved to be at all close to a "gold standard" for overuse patellar tendon injury. Lian and his Norwegian colleagues ${ }^{14}$ first showed that US lacked both sensitivity and specificity against the clinical diagnosis of jumper's knee in volleyball players. US does not show tendon abnormalities in patients with clinically diagnosed jumper's knee, ${ }^{14}$ and it can remain normal in areas proved by histopathological examination to contain significant tendon degeneration (tendinosis). ${ }^{16}$ Conversely, US abnormalities exist in athletes with no current or previous anterior knee pain. ${ }^{14}{ }^{17-19}$ Recently, Shalaby and Almekinders ${ }^{15}$ showed similar limitations of MRI of the patellar tendon and used clinical assessment as the diagnostic standard saying that "symptoms and findings of patellar tendinitis are generally simple to elicit and are usually not the result of other conditions around the knee". Postoperative imaging has also shown lack of correlation between symptoms and both US and MRI findings on the tendon. ${ }^{20}{ }^{21}$ Thus the existence of patients with "imaging-normal" jumper's knee cannot be disputed. ${ }^{22}$ Therefore, like Shalaby and Almekinders, ${ }^{15}$ we used expert clinical assessment for diagnosis. 
EXERCISE TREATMENT FOR JUMPER'S KNEE

We found that a 12 week programme of either drop squats or leg extension/curl exercises was associated with pain reduction. One practical advantage of drop squats over leg extension/ curls, if they are equally effective, is that drop squats can be performed without any special strength training equipment. The hand held weights required for drop squats can be made by filling household containers with water and weighing them on a kitchen scale. Although subjects in this research subject complied with the leg extension/curl exercise programme, not all patients would have immediate access to the necessary equipment.

To our knowledge, there have only been two studies of exercise treatment for jumper's knee that measured clinical outcome, ${ }^{78}$ and both were uncontrolled. In their seminal tendinopathy monograph, Stanish and Curwin ${ }^{7}$ report complete relief of symptoms in one of three patients with jumper's knee and moderate relief in the other two thirds, all of whom undertook six to eight weeks of drop squat training. We based our drop squat prescription on this work. Karlsson et $^{\mathrm{a}} \mathrm{l}^{8}$ reported that $70 \%$ of 81 patients with jumper's knee had excellent outcomes after a combined concentric and eccentric rehabilitation protocol. One placebo controlled trial of eccentric isokinetic strengthening measured strength changes but not pain or return to activity. ${ }^{9}$

Although our small study did not detect a difference between the effect of the drop squats and the leg extension/curl exercises, both randomised ${ }^{10}$ and non-randomised ${ }^{11}$ studies of exercise treatment in Achilles tendinopathy have emphasised the importance of eccentric training.

We note that, although a proportion $(4 / 10)$ of patients who undertook the drop squat programme became pain free within three months, a further five were improved but continued to rate symptoms at about 3 of 10 , indicating that this condition does not always improve in three months. Studies with 12 months or longer follow up are needed.

There was no placebo group, as we felt it unreasonable to deny patients any form of strength training. Thus it could be argued that our data merely reflect the natural history of jumper's knee. Several pieces of evidence dispute that assertion. Firstly, a large study of athletes with jumper's knee found that $69 \%$ of subjects required in excess of 12 weeks out of sport despite a range of conservative treatments that included the type of strength training used in this study. ${ }^{23}$ Half of those subjects required more than six months out of sport. Secondly, the large number of patients who have had patellar tendon surgery after failed conservative management ${ }^{5}$ also indicates that the condition is not self limiting. Lastly, clinical experience suggests that a 12 week recovery from jumper's knee is a good result.

We recommend that future randomised controlled trials of treatment for jumper's knee should include a group prescribed corticosteroid/non-steroid anti-inflammatory drugs as well as an exercise treatment group.
Although the biological mechanism underpinning the efficacy of corticosteroid injections remains unknown, ${ }^{24}$ their effectiveness in the rotator cuff ${ }^{25}$ and the lateral elbow ${ }^{26}$ tendons and clinical experience ${ }^{27}$ suggest at least temporary benefit in jumper's knee.

FACTORS ASSOCIATED WITH PAIN REDUCTION We examined whether demonstrable muscle weakness, as measured by isokinetic dynamometry, was directly linked with the pain of jumper's knee. We found that hamstring weakness did not contribute to this conditioncertainly not when tested at $30 \%$ second. With respect to quadriceps strength, the drop squat group increased strength by $15 \%$ and the leg extension/curl group gained no strength. Unfortunately, the significant difference between groups at baseline prevents us from drawing conclusions as to the relative efficacy of the two programmes. Future studies should evaluate this question further, at a variety of speeds of knee extension, and perhaps using functional tests such as jump tests. The source of pain in tendinopathies remains unclear. ${ }^{28-30}$

STUDY STRENGTHS AND LIMITATIONS

To our knowledge, this small study is the first report of a randomised clinical trial of strengthening exercises in jumper's knee. The exercise protocol was clearly documented and compliance was excellent in the largely university based study population. No confounding treatments were given-no subjects used orthotics or physiotherapy modalities. No subjects used medication after the first two weeks. All patients were assessed by only three clinicians who are extremely experienced sports medicine specialists. Data collection by workers blinded to subjects' treatment group prevented a potential bias. ${ }^{5}$

Because the subject population was small, there was limited power to detect differences in outcome between the two types of training. Also, the subject population was rather heterogeneous: both sexes were included and there was a wide age range. This was necessary to obtain sufficient numbers because jumper's knee is not a very common condition and use of orthotics was an exclusion criterion. As discussed above, the absence of a true "control" group is a limitation. We note that a recent study of soccer players with groin pain (not necessarily adductor tendon pain, but possibly including it) precluded the control group from strength exercises and only 3\% of that group returned to sport in six months. ${ }^{31}$ As our clinic provides a service to athletes, we sought to answer the clinically relevant question without compromising athlete care.

In summary, we found that a drop squat programme was generally safe in patients with jumper's knee and that it reduced pain effectively, as did a leg extension/curl programme. These findings support studies performed in other major tendons - for example, Achilles tendon, lateral elbow tendon-that indicate the effectiveness of strengthening exercise programmes. ${ }^{10}{ }^{11}$ On the other hand, our data also support the contention that 
jumper's knee is a challenging condition for clinicians, as only six of $19(32 \%)$ subjects were completely pain free at 12 weeks. We conclude that, at present, there is no guaranteed rapid conservative cure for jumper's knee, and surgical outcomes are unpredictable. ${ }^{532}$ We recommend further trials comparing various strengthening protocols with each other and with other treatments (such a corticosteroid/ anaesthetic injections), and strongly encourage multicentre collaboration to increase the power of studies.

1 Blazina ME, Kerlan RK, Jobe FW, et al. Jumper's knee. Orthop Clin North Am 1973;4:665-78.

2 Maffulli N, Khan KM, Puddu G. Overuse tendon conditions. Time to change a confusing terminology. conditions. Time to chan

Arthroscopy 1998;14:840-3.
3 Khan KM, Cook JL, Bonar F, et al. Histopathology of comKhan KM, Cook JL, Bonar F, et al. Histopathology of common overuse tendon conditions: update and implication

4 Cook JL, Khan KM, Maffulli N, et al. Patellar tendinopathy: new approaches to a chronic problem. The physician and sports medicine 2000;28:31-46.

5 Coleman BD, Khan KM, Maffulli N, et al. Studies of surgical outcome after patellar tendinopathy: clinical significance of methodological deficiencies and guidelines for future studies. Scand F Med Sci Sports 2000;10:2-11.

6 Khan KM, Maffulli N. Tendinopathy: an Achilles' heel for athletes and clinicians. [Lead editorial] Clin 7 Sport Med 1998;8:151-4.

7 Stanish WD, Curwin KS. Tendinitis. Its etiology and treatment. Toronto: Collamore Press, 1984.

8 Karlsson J, Kalebo P, Goksor L-A, et al. Partial rupture of the patellar ligament. Am $\mathcal{f}$ Sports Med 1992;20:390-5.

9 Jensen K, Di Fabio RP. Evaluation of eccentric exercise in treatment of patellar tendinitis. Phys Ther 1989;69:211-16.

10 Niesen-Vertommen SL, Taunton JE, Clement DB, et al. The effect of eccentric versus concentric exercise in the effect of eccentric versus concentric exercise in the management

11 Alfredson H, Pietila T, Jonsson P, et al. Heavy-load eccentric calf muscle training for the treatment of chronic Achilles tendinosis. Am F Sports Med 1998;26:360-6.

12 Fritschy D, de Gautard R. Jumper's knee and ultrasonography. Am f Sports Med 1988;16:637-40.

3 Myllymäki T, Bondestam S, Suramo I, et al. Ultrasonography of jumper's knee. Acta Radiol 1990;31:147-9.

14 Lian O, Holen KJ, Engebrestson L, et al. Relationship between symptoms of jumper's knee and the ultrasound characteristics of the patellar tendon among high level male volleyball players. Scand 7 Med Sci Sports 1996;6:291-6.

15 Shalaby M, Almekinders LC. Patellar tendinitis: the significance of magnetic resonance imaging findings. Am $\mathcal{F}$ Sports Med 1999;27:345-9.
16 Movin T, Gad A, Reinholt FP, et al. Tendon pathology in long-standing achillodynia. Biopsy findings in 40 patients. Acta Orthop Scand 1997;68:170-5.

17 Cook JL, Khan KM, Harcourt PR, et al. Patellar tendon ultrasonography in asymptomatic active athletes reveals hypoechoic regions: a study of 320 tendons. Clin f Sport Med 1998;8:73-7.

18 Cook JL, Khan KM, Kiss ZS, et al. Prospective imaging study of asymptomatic patellar tendinipathy in elite junior basketball players. F Ultrasound Med 2000;19:473-9.

19 Cook JL, Khan KM, Kiss ZS, et al. Patellar tendinopathy in junior basketball players: a controlled clinical and ultrasonographic study of 268 patellar tendons in players aged 14-18 years. Scand F Med Sci Sports 2000;10:216-20.

20 Kiss ZS, Kellaway D, Cook J, et al. Postoperative patellar tendon healing: an ultrasound study. Australas Radiol 1998; 42:28-32.

21 Khan KM, Visentini PJ, Kiss ZS, et al. Correlation of US and MR imaging with clinical outcome after open patellar tenotomy: prospective and retrospective studies. Clin $\mathcal{F}$ Sport Med 1999;9:129-37.

22 Cook JL, Kiss ZS, Khan KM. Patellar tendinitis: the significance of magnetic resonance imaging findings [letter]. Am 7 Sports Med 1999;27:831.

23 Cook JL, Khan K, Harcourt PR, et al. A cross-sectional study of 100 cases of jumper's knee managed conservatively and surgically. Br $\mathcal{F}$ Sports Med 1997;31:332-6.

24 Kraushaar BS, Nirschl RP. Tendinosis of the elbow (tennis elbow). Clinical features and findings of histological, mmunohistochemical, and electron microscopy studies. $\mathcal{f}$ Bone foint Surg [Am] 1999;81:269-78.

25 Blair B, Rokito AS, Cuomo F, et al. Efficacy of injections of corticosteroids for subacromial impingement syndrome. $\mathcal{F}$ Bone foint Surg $[\mathrm{Am}]$ 1996;78:1685-9.

26 Hay EM, Paterson SM, Lewis $M$, et al. Pragmatic randomised controlled trial of local corticosteroid injection and naproxen for treatment of lateral epicondylitis of elbow in primary care. BMF 1999;319:964-8.

27 Józsa L, Kannus P. Human tendons. Champaign, IL: Human Kinetics, 1997:576.

28 Alfredson H, Thorsen K, Lorentzon R. In situ microdialysis in tendon tissue: high levels of glutamate, but not prostaglandin E2 in chronic achilles tendon pain. Knee Surg Sports Traumatol Arthrosc 1999;7:378-81.

29 Khan KM, Cook JL, Maffulli N, et al. Where is the pain coming from in tendinopathy? It may be biochemical, not only structural, in origin. Br F Sports Med 2000;34:81-4.

30 Khan KM, Cook JL. Overuse tendon injuries: where does the pain come from? Sports Medicine and Arthroscopy Reviews 2000;8:17-31.

31 Holmich P, Uhrskou P, Ulnits L, et al. Effectiveness of active physical training as treatment for long-standing adductorrelated groin pain in athletes: randomised trial. Lancet 1999;353:439-53.

32 Coleman BD, Khan KM, Kiss ZS, et al. Outcomes of open and arthroscopic patellar tenotomy for chronic patellar tendinopathy: a retrospective study. Am F Sports Med 2000; 28:183-90. 